\title{
La métrologie des rayonnements neutroniques à l'IRSN
}

\author{
V. LACOSTE ${ }^{1}$, C. MONNIN-PARIETTI ${ }^{1}$
}

(Manuscrit reçu le 6 novembre 2006, accepté le 15 février 2007)

RÉSUMÉ L'Institut de radioprotection et de sûreté nucléaire détient les références nationales pour la fluence et les grandeurs dosimétriques neutroniques. Ces références, maintenues et exploitées depuis une dizaine d'années, sont le fruit d'une volonté de développer des installations neutroniques de qualité permettant de caractériser des instruments de radioprotection et de dosimétrie de manière fiable. En effet, que ce soit pour ses propres besoins, du fait de ses activités de recherche et développement d'appareils de mesures en radioprotection ou pour des utilisateurs externes, l'IRSN a développé un ensemble de dispositifs et d'installations permettant de répondre aux recommandations des instances internationales normatives quant aux procédures et aux moyens d'irradiation nécessaires pour caractériser les instruments de radioprotection.

ABSTRACT Neutron metrology at IRSN.

The Institute for Radiological Protection and Nuclear Safety owns the french references for the neutron fluence and dosimetric quantities. These references, which are maintained and exploited since a tenth of years, are the result of the willingness to develop neutron facilities of quality, permitting to characterize survey instruments and dosimeters in a reliable way. Indeed, for its own needs regarding its activities related to the research and development of radiation protection instruments, or for external users, the IRSN has developed a set of facilities which allows to follow the recommendations of the international commission for standardization regarding the procedures and means of irradiations requested to characterize the survey instruments.

Keywords: neutron fields / accelerators / neutron calibration

\section{Historique}

Les activités de l'IRSN en matière de production de rayonnements neutroniques se sont développées initialement pour répondre aux besoins d'étalonnage des systèmes de dosimétrie individuelle, développés dans les années 50-60 par le CEA. Les premiers champs de rayonnements neutroniques étaient produits par des accélérateurs permettant de disposer de neutrons de $3 \mathrm{MeV}$, sur les sites respectifs

${ }^{1}$ IRSN, Service de dosimétrie externe, laboratoire de métrologie et de dosimétrie des neutrons, BP 3, 13115 Saint-Paul lez Durance, France. 
de Fontenay-aux-Roses puis de Cadarache où un empilement de graphite contenant des sources de ${ }^{241} \mathrm{Am}-\mathrm{Be}$ était également disponible pour des irradiations en champ à forte composante thermique.

En 1990, les nouvelles recommandations de la Commission internationale de protection radiologique (ICRP, 1990) impliquèrent la nécessité d'améliorer la limite de détection des dosimètres individuels, leur réponse angulaire et énergétique. Les nouvelles techniques de dosimétrie individuelle, comptant parmi elles les systèmes électroniques, demandèrent de nouveaux moyens d'essai et d'étalonnage appropriés. Pour répondre à ces nouveaux besoins, un ensemble de travaux a été élaboré, portant notamment sur les rayonnements de référence avec le programme METHOD, mis en place par J.-L. Chartier. Ce programme de recherche comportait trois volets, concernant les dispositifs de production de champs neutroniques, les systèmes spectrométriques d'analyse des champs de rayonnements, et les procédures d'étalonnage des systèmes dosimétriques individuels et d'environnement. L'ensemble des actions liées au programme de développement des champs neutroniques de référence, ces derniers n'existant pas par ailleurs en France, était soutenu par le Bureau national de métrologie (BNM), avec lequel une convention de recherche a été signée à cette époque. Le laboratoire de dosimétrie externe du service de dosimétrie (LRDE/SDOS), en marge de ces travaux de recherche, assurait le maintien des références neutroniques déjà existantes, correspondant aux sources de ${ }^{241} \mathrm{AmBe},{ }^{252} \mathrm{Cf}$ et de ${ }^{252} \mathrm{Cf}$ modérée par $\mathrm{D}_{2} \mathrm{O}$, pour lesquelles le laboratoire avait une habilitation $\mathrm{SMH}$ (Service de métrologie habilité).

Le programme de recherche relatif au développement de nouveaux champs neutroniques de référence pour l'étalonnage des instruments de radioprotection était principalement motivé par le fait que l'ensemble des spectres de référence recommandés par la norme ISO (4 spectres) n'était pas suffisamment représentatif des différentes situations rencontrées sur le terrain. L'idée innovante de ce programme était donc de compléter les moyens d'étalonnage par d'autres dispositifs délivrant des spectres neutroniques plus variés et mieux adaptés aux situations observées auprès des postes de travail de l'industrie nucléaire. C'est ainsi qu'en 1991, le dispositif CANEL+ (Chartier, 1991), composé d'un convertisseur en ${ }^{238} \mathrm{U}$ et d'un canal modérateur en polyéthylène, a été mis en place afin de produire en laboratoire des champs neutroniques réalistes.

Ce dispositif modulaire, installé sur le site de Cadarache, était utilisé initialement avec un accélérateur SAMES $150 \mathrm{kV}$ délivrant des neutrons rapides de $14 \mathrm{MeV}$, puis en 1994, avec un SAMES $400 \mathrm{kV}$, délivrant des neutrons de $3 \mathrm{MeV}$. 
En marge des étalonnages de routine auprès de sources radioactives et des champs réalistes, la caractérisation fine de la réponse en fonction de l'énergie des instruments de radioprotection était tout autant nécessaire en raison des variations importantes des coefficients de conversion fluence à dose (ICRU, 1998). Après des travaux relatifs à l'établissement de références neutroniques monocinétiques disponibles sur l'une des lignes du Van de Graaf de Bruyères-le-Châtel dans les années 90, le laboratoire décida de se doter d'un accélérateur permettant de produire des champs monocinétiques pour répondre à ses besoins croissants en matière de caractérisation de ses propres instruments de référence et de radioprotection. L'installation AMANDE, délivrant des champs neutroniques d'énergie comprise entre $2 \mathrm{keV}$ et $20 \mathrm{MeV}$, est opérationnelle depuis 2005 et ouverte aux utilisateurs externes.

Aujourd'hui, le laboratoire de métrologie et de dosimétrie des neutrons possède un plateau technique complet pour la production de champs neutroniques recommandés par les normes ISO pour la caractérisation des instruments de radioprotection. Depuis 1997, les activités d'étalonnage auprès des sources radioactives sont reconnues par le BNM (intégré depuis dans le Laboratoire national de métrologie et d'essais - LNE) par le biais d'un contrat de « laboratoire associé », et d'une accréditation COFRAC. Le laboratoire compte parmi ses objectifs à moyen terme la labellisation de l'ensemble de ses installations (SAMES 150kV, SAMES 400 kV, CANEL, SIGMA et AMANDE).

\section{Les champs neutroniques de référence}

Les champs neutroniques de référence sont définis dans les normes de l'Organisation internationale de normalisation (International Standard Organisation - ISO) qui se réfèrent elles-mêmes aux normes de la Commission electrotechnique internationale (CEI) relatives aux instruments et équipements. Divers types de champs sont recommandés selon les applications qui peuvent être soit des étalonnages de routine d'instruments de radioprotection ou de dosimètres personnels, soit des études et caractérisations fines des instruments de spectrométrie, de radioprotection ou de dosimétrie à des énergies données.

Dans le premier cas, pour l'étalonnage de routine de dispositifs de mesure ayant déjà été soumis à l'ensemble des tests de fonctionnement sous rayonnement lors de leur conception ou homologation, les sources radioactives ${ }^{241} \mathrm{AmBe}$ (neutrons produits par réaction $(\alpha, \mathrm{n})$ ) et ${ }^{252} \mathrm{Cf}$ (neutrons issus de la fission spontanée du radio-nucléide) sont recommandées par la norme ISO 8529-1 (2001). L'énergie moyenne des neutrons (4,2 MeV pour AmBe et $2,1 \mathrm{MeV}$ pour ${ }^{252} \mathrm{Cf}$ ) est du même ordre que celle des neutrons produits par réaction de fission dans le cycle du combustible. 
Les dosimètres individuels et, en général, tous les appareils de mesure des neutrons ont une réponse dépendant de l'énergie des neutrons. Il peut donc s'avérer impossible d'étalonner correctement un instrument qui sera utilisé dans un champ neutronique dont les distributions en énergie et angulaire diffèrent significativement du champ de rayonnement de référence utilisé pour l'étalonnage. C'est à cet effet que la norme ISO 12789-2 (2000) a introduit la notion de « champs neutroniques simulant ceux de postes de travail », également appelés «champs neutroniques réalistes». Ces champs neutroniques, une fois précisément caractérisés, peuvent être utilisés comme champs de référence. Ils sont également recommandés dans les nouvelles normes de la CEI. L'objectif est de pouvoir étalonner tout appareil dans un champ neutronique aussi proche que possible de celui dans lequel il sera utilisé. L'étalonnage dans de tels champs réalistes permet d'obtenir une dosimétrie plus fiable et plus adaptée à chaque poste de travail.

L'étude de la variation de la réponse des instruments de mesure avec l'énergie des neutrons permet de valider les réponses neutroniques calculées des instruments et/ou de déterminer et de vérifier l'adéquation des moyens d'étalonnage de ces instruments.

Pour réaliser une telle étude, les champs neutroniques utilisés doivent avoir une distribution en énergie limitée sur une plage étroite en énergie. Les champs neutroniques recommandés sont :

- les champs de neutrons thermiques, produits généralement auprès de réacteurs, et dont l'énergie est inférieure à $0,5 \mathrm{eV}$,

- les champs de neutrons mono-énergétiques ou quasi mono-énergétiques produits par faisceau filtré de réacteur $(2 \mathrm{keV}, 24 \mathrm{keV}$ et $144 \mathrm{keV})$ ou par réactions nucléaires à l'aide d'accélérateurs de particules chargées. Les réactions nucléaires utilisées sont détaillées dans le paragraphe 3.

\section{Les sources radioactives de référence}

L'IRSN dispose de sources radioactives ${ }^{241} \mathrm{AmBe}$ et ${ }^{252} \mathrm{Cf}$ (Van Ryckeghem et al., 2002) recommandées par la norme ISO 8529-1 pour les étalonnages de routine. Ces sources sont utilisées « nues », mais afin de produire des champs « réalistes », et selon les recommandations de cette même norme, la source de ${ }^{252} \mathrm{Cf}$ peut être placée au centre d'une sphère modératrice d'eau lourde de $15 \mathrm{~cm}$ de rayon recouverte ou non d'une coquille de cadmium. La configuration sans cadmium n'est pas recommandée par l'ISO mais elle représente également un champ réaliste, avec une composante thermique. 
TABLEAU I

Fluence et équivalent de dose (ambiant et individuel) à $75 \mathrm{~cm}$ de la source.

Fluence and dose equivalent rates (ambient and personal) at $75 \mathrm{~cm}$ from the source.

\begin{tabular}{ccccc}
\hline & ${ }^{241} \mathrm{Am}-\mathrm{Be}$ & ${ }^{252} \mathrm{Cf}$ & $\left({ }^{252} \mathrm{Cf}+\mathrm{D}_{2} \mathrm{O}\right)_{/ \mathrm{Cd}}$ & ${ }^{252} \mathrm{Cf}+\mathrm{D}_{2} \mathrm{O}$ \\
\hline Emission $\left(\mathrm{s}^{-1}\right)$ & $4 \times 10^{7}$ & $3 \times 10^{8}$ & $3 \times 10^{8}$ & $3 \times 10^{8}$ \\
$\dot{\Phi}_{\mathrm{n}}\left(\mathrm{cm}^{-2} \cdot \mathrm{s}^{-1}\right)$ & $505 \pm 8$ & 4300 & 3700 & 3900 \\
$\dot{H}^{*}(10)\left(\mu{\left.\mathrm{Sv} \cdot \mathrm{h}^{-1}\right)}^{711 \pm 31}\right.$ & 5900 & 1400 & 1400 \\
$\dot{H}_{\mathrm{p}}\left(10,0^{\circ}\right)\left(\mu{\left.\mathrm{Sv} \cdot \mathrm{h}^{-1}\right)}^{747 \pm 32}\right.$ & 6100 & 1500 & 1500 \\
\hline
\end{tabular}

Au-delà de l'activité d'étalonnage de routine, cet irradiateur, dénommé « Van Gogh » a pour mission de raccorder les laboratoires accrédités aux références nationales pour la dosimétrie des neutrons. Dans ce cadre, les activités d'étalonnage à l'aide de cet irradiateur sont accréditées par le COFRAC (Comité français d'accréditation).

Les débits de fluence et les débits d'équivalent de dose (individuel et ambiant) à $75 \mathrm{~cm}$ des sources, à la date du $1^{\mathrm{er}}$ mai 2005, sont présentés dans le tableau I (Gressier, 2006). Pour la source de ${ }^{252} \mathrm{Cf}$, les valeurs sont des estimations issues de l'activité attendue de la nouvelle source installée dans l'irradiateur en avril 2005.

\section{Les installations produisant des champs mono-énergétiques}

L'IRSN possède trois accélérateurs permettant de générer des champs neutroniques mono-énergétiques (Gressier, 2006).

Deux d'entre eux sont des générateurs de neutrons de type SAMES, appelés communément $\mathrm{J} 25$ et T400, accélérant des deutons à des énergies maximales de $120 \mathrm{keV}$ et $350 \mathrm{keV}$ respectivement. Ces deutons accélérés sont envoyés sur des cibles de titane tritié (accélérateur J25) ou de titane deutérié (accélérateur T400) où leur interaction avec les atomes de tritium ou de deutérium génère des champs de neutrons quasi mono-énergétiques d'énergie moyenne respectivement de 3,1 MeV et 14,7 MeV. La fluence neutronique est déterminée et monitorée en utilisant la méthode de la «particule associée » : trois diodes silicium sont placées à l'arrière de la cible (angle de $175^{\circ}$ ) et détectent les particules alpha issues de la réaction ${ }^{3} \mathrm{H}(\mathrm{d}, \mathrm{n}){ }^{4} \mathrm{He}(\mathrm{J} 25)$ ou les protons émis lors de la réaction ${ }^{2} \mathrm{H}(\mathrm{d}, \mathrm{p})^{3} \mathrm{H}$, réaction compétitive à la réaction produisant les neutrons ${ }^{2} \mathrm{H}(\mathrm{d}, \mathrm{n})^{3} \mathrm{He}(\mathrm{T} 400)$.

Lors des mesures, les appareils peuvent être placés à une distance de la cible comprise entre 0 et $2 \mathrm{~m}$, centrés dans l'axe du faisceau de deutons $\left(0^{\circ}\right)$. Les principales caractéristiques des champs neutroniques produits auprès de ces deux 
TABLEAU II

Caractéristiques des champs neutroniques produits auprès des accélérateurs $\mathbf{T} 400$ et $\mathbf{J} 25$, dans les conditions nominales de fonctionnement.

Characteristics of the neutron fields produced by the T400 and J25 accelerators for nominal running conditions.

\begin{tabular}{ccccccc}
\hline Accélérateur & $\begin{array}{c}\text { Énergie des } \\
\text { deutons }\end{array}$ & $\begin{array}{c}E \mathrm{n}\left(0^{\circ}\right) \\
(\mathrm{MeV})\end{array}$ & $\Delta E \mathrm{n} / \mathrm{En}$ & $\begin{array}{c}\text { Émission } \\
\text { neutronique }\left(\mathrm{s}^{-1}\right)\end{array}$ & $\begin{array}{c}\mathrm{d} \Phi / \mathrm{dt} \max \\
\left(\times 10^{4} \mathrm{~cm}^{-2} \cdot \mathrm{s}^{-1}\right)\end{array}$ & $\begin{array}{c}\mathrm{d} H \mathrm{p}\left(10,0^{\circ}\right) / \mathrm{dt} \max \\
(\mathrm{mSv} / \mathrm{h})\end{array}$ \\
\hline $\mathrm{T} 400$ & $350 \mathrm{keV}$ & 3,1 & $10 \%$ & $2,2 \times 10^{9}$ & $3,6 \pm 0,2$ & $56 \pm 3$ \\
$\mathrm{~J} 25$ & $120 \mathrm{keV}$ & 14,7 & $1,7 \%$ & $1,4 \times 10^{10}$ & $11,8 \pm 0,7$ & $240 \pm 13$ \\
\hline
\end{tabular}

accélérateurs sont données dans le tableau II. La résolution en énergie neutron $\Delta E \mathrm{n}$ correspond à la largeur à mi-hauteur du pic «mono-énergétique ». Les valeurs maximales des débits de fluence et débits d'équivalent de dose individuel sont données pour une distance de $1 \mathrm{~m}$ par rapport à la cible, dans l'axe du faisceau de deutons et correspondent à 3000 coups/s et 2400 coups/s respectivement dans les diodes du T400 et du J25.

Ces deux accélérateurs sont utilisés pour des applications dosimétriques et de radioprotection qui demandent le plus souvent des débits de fluence élevés et une résolution en énergie moins fine que ce que peut fournir le troisième accélérateur, AMANDE, mis en service en 2005, dédié principalement aux applications métrologiques.

AMANDE est un tandetron de $2 \mathrm{MV}$, c'est-à-dire un accélérateur de type Cockroft Walton de géométrie tandem, permettant de couvrir le plus grand domaine possible en énergie de neutrons mono-énergétiques avec un accélérateur de taille raisonnable. AMANDE permet d'accélérer en continu ou en mode pulsé des protons et des deutons à des énergies comprises entre $100 \mathrm{keV}$ et $4 \mathrm{MeV}$ avec une excellente résolution et une très grande stabilité en énergie $(<500 \mathrm{eV})$. Les champs neutroniques sont obtenus à partir de particules chargées (protons et deutons) accélérées puis dirigées sur une cible constituée d'un dépôt de scandium, lithium, tritium, ou deutérium sur un mince support métallique. L'interaction des particules chargées avec les noyaux de ces éléments génère des neutrons dans tout l'espace. Les réactions nucléaires correspondantes produisent des neutrons d'une seule énergie pour une énergie donnée de particule chargée incidente et un angle d'émission donné des neutrons. Les énergies de neutrons produits à $0^{\circ}$ par rapport à l'axe du faisceau, en fonction des différentes particules incidentes, sont les suivantes :

- ${ }^{45} \mathrm{Sc}(\mathrm{p}, \mathrm{n})$ pour les énergies $2 \mathrm{keV}$ et $24 \mathrm{keV}$ recommandées par la norme ISO ;

- ${ }^{7} \mathrm{Li}(\mathrm{p}, \mathrm{n})$ pour les énergies $144 \mathrm{keV}, 250 \mathrm{keV}$ et $565 \mathrm{keV}$;

- ${ }^{3} \mathrm{H}(\mathrm{p}, \mathrm{n})$ pour les énergies $1,2 \mathrm{MeV}$ et $2,5 \mathrm{MeV}$; 
- ${ }^{2} \mathrm{H}(\mathrm{d}, \mathrm{n})$ pour les énergies $2,8 \mathrm{MeV}$ et $5 \mathrm{MeV}$;

- ${ }^{3} \mathrm{H}(\mathrm{d}, \mathrm{n})$ pour les énergies de $14,8 \mathrm{MeV}$ et $19 \mathrm{MeV}$.

Les caractéristiques des faisceaux mono-énergétiques produits sont détaillées dans l'article de Gressier (2006).

L'accélérateur AMANDE devrait en outre permettre de produire des rayonnements gamma autour de $7 \mathrm{MeV}$ d'énergie par la réaction ${ }^{19} \mathrm{~F}(\mathrm{p}, \alpha \gamma){ }^{16} \mathrm{O}$. Cette source de rayonnement complétera les moyens d'irradiation par photons de l'IRSN dont l'énergie maximale est actuellement de $1,32 \mathrm{MeV}$ (source de ${ }^{60} \mathrm{Co}$ ).

\section{Les installations produisant des champs neutroniques réalistes}

«CANEL » (CAnon à NEutron Lent) est un dispositif modulaire qui vient se placer autour de la cible des générateurs de neutrons J25 et T400 pour produire des champs neutroniques « réalistes » (Gressier, 2006). C'est un dispositif unique au monde cité à titre d'exemple dans la norme ISO 12789.

Le principe de CANEL est de générer un spectre de neutrons de fission et de le modérer à l'aide d'éléments représentatifs des dispositifs de modération des neutrons mis en place autour des réacteurs de l'industrie nucléaire. Les neutrons quasi mono-énergétiques issus des accélérateurs rencontrent tout d'abord une coquille d'uranium appauvri, dans laquelle ils sont convertis par réaction de fission.

Ces neutrons de fission traversent ensuite des écrans de fer puis de polyéthylène, le tout étant confiné dans un canal en polyéthylène de $70 \mathrm{~cm}$ de diamètre et de $1 \mathrm{~m}$ de longueur. La taille des écrans peut varier selon l'accélérateur utilisé (J25 ou T400). Le point d'étalonnage des appareils est situé dans l'axe du faisceau de deutons à $30 \mathrm{~cm}$ de la sortie de CANEL dans la configuration CANEL/J25 et $50 \mathrm{~cm}$ dans la configuration CANEL/T400. La configuration du dispositif CANEL avec un écran modérateur en polyéthylène est en phase de caractérisation auprès de l'accélérateur T400, alors qu'une nouvelle configuration est en cours d'étude pour son couplage avec le J25 pour s'affranchir au maximum des neutrons de $14 \mathrm{MeV}$, lesquels contribuaient de manière trop importante à la dose dans l'ancienne configuration utilisant une lentille d'eau. De manière générale, un ensemble de configurations est en cours d'étude afin de répondre au mieux aux caractéristiques des spectres rencontrés auprès d'installations de l'industrie nucléaire. Ces configurations, adaptées aux postes de travail auprès desquels des mesures de spectrométrie neutronique ont été réalisées au cours des dernières années, offriront aux exploitants la possibilité de caractériser et 
d'étalonner leurs instruments de radioprotection et leurs dosimètres personnels auprès de champs de référence similaires à ceux existants dans leurs installations.

\section{Conclusions}

L'IRSN offre un plateau technique complet pour produire tous les types de champ neutronique recommandés par les normes internationales pour l'étalonnage de tout instrument de détection des neutrons. L'ensemble des installations est ouvert à tout utilisateur.

Dans le cadre de la norme ISO 12789-1 recommandant l'étalonnage des instruments de radioprotection et des dosimètres personnels auprès de champs réalistes adaptés aux postes de travail auprès desquels ces appareils seront ensuite utilisés, différentes configurations du dispositif CANEL sont en cours d'étude. Les configurations devront répondre au mieux aux caractéristiques des champs rencontrés par exemple aux postes de travail de diverses installations industrielles nucléaires, caractérisés lors du programme européen EVIDOS (BologneseMilsztajn et al., 2004).

\section{RÉFÉRENCES}

Bolognese-Milsztajn T., Bartlett D., Boschung M., Coeck M., Curzio G., d'Errico F., Fiechtner A., Giusti V., Gressier V., Kyllonen J., Lacoste V., Lindborg L., Luszik-Bhadra M., Molinos C., Pelcot G., Reginatto M., Schuhmacher H., Tanner R., Vanhavere F., Derdau D. (2004) Individual neutron monitoring in workplaces with mixed neutron/photon radiation, Rad. Prot. Dosim. 110, 753-758.

Chartier J.-L., Posny F., Buxerolle M. (1991) Experimental assembly for the simulation of realistic neutron spectra, Rad. Prot. Dosim. 44, 125-130.

Gressier V. (2006) Les installations de l'IRSN dédiées à la métrologie des neutrons, Radioprotection 41, 11-32.

ICRP Publication 60 (1990) Recommendations of the International Commission on Radiological Protection.

ICRU Report 57 (1998) Conversion coefficients for use in Radiological Protection Against External Radiation.

Norme ISO 8529-1 (2001) Rayonnements neutroniques de référence - Partie 1 : Caractéristiques et méthodes de production.

Norme ISO 12789-1 (2000) Rayonnements neutroniques de référence, Caractéristiques et méthodes de production de champs de neutrons simulant ceux de postes de travail.

Van Ryckeghem L., Lacoste V., Pelcot G., Pochat J.-L. (2002) Neutron calibration facilities of the IRSN research laboratory in external dosimetry. In: Proceeding of the 11th International Symposium on Reactor Dosimetry, 18-23 August, 2002, Brussels, pp. 528-535. 